ARTICLE OPEN

\title{
Inflammatory gene expression signatures in idiopathic intracranial hypertension: possible implications in microgravity-induced ICP elevation
}

\author{
Susana B. Zanello ${ }^{1}$, Vasisht Tadigotla², James Hurley², Johan Skog ${ }^{2}$, Brian Stevens ${ }^{3}$, Eusebia Calvillo ${ }^{3}$ and Eric Bershad ${ }^{3}$
}

The visual impairment and intracranial pressure (VIIP) syndrome is a neuro-ophthalmologic condition described in astronauts returning from long duration space missions. Idiopathic intracranial hypertension $(\mathrm{IIH})$, also known as pseudotumor cerebri, is characterized by a chronic elevation of intracranial pressure (ICP) in the absence of an intracranial mass lesion. Because VIIP and IIH share some neurologic and ophthalmologic manifestations, the latter might be used as a model to study some of the processes underlying VIIP. This work constitutes a preliminary investigation of the molecular pathways associated with the elevation of ICP in $\mathrm{IIH}$. Gene expression signatures were obtained from exosomes collected from CSF and plasma in patients with possible signs of IIH. The gene expression targets focused on inflammatory genes and miRNAs. The results suggest that inflammatory cytokine-driven processes and immune cell migration are activated when ICP is elevated in IIH patients, either as a cause or effect of the ICP increase. Several miRNAs appear to be involved in this response, among which miR-9 and miR-16 are upregulated in CSF and plasma of higher ICP subjects. This study provides evidence in support of neurophysiological alterations and neuroimmunomodulation in this condition. If similar changes are seen in astronauts manifesting with the VIIP syndrome, an underlying pathophysiological basis may be discovered.

npj Microgravity (2018)4:1 ; doi:10.1038/s41526-017-0036-6

\section{INTRODUCTION}

Human space exploration involves multi-system health risks. Neuro-ophthalmologic symptoms, including elevated intracranial pressure (ICP) upon return to Earth, ${ }^{1}$ have been observed in astronauts participating in long-duration missions, a condition named visual impairment and intracranial pressure syndrome (VIIP). On Earth, these neuroanatomical findings concur with those in idiopathic intracranial hypertension $(\mathrm{IIH})^{2}$ The etiology of $\mathrm{IH}$ is a topic of debate. The moderately elevated ICP over many years has been linked to cognition losses relieved by lumbar tapping to reduce ICP. ${ }^{3,4}$ While most cases are seen in overweight women of child-bearing age, there is no specific condition on Earth that shares more similarities to VIIP, constituting a reasonable analog to study the effects of chronically elevated ICP at the molecular level.

A number of performance ${ }^{5-7}$ and neurologic signs ("space $\left.\mathrm{fog}^{\prime \prime}\right)^{8}$ have also been linked to spaceflight and might also result from elevation of ICP. In this paper, we test the hypothesis that these symptoms are caused by disturbances in the neurophysiology of the brain and correlated with molecular markers in the cerebrospinal fluid (CSF) by studying gene expression profiles from CSF and plasma in individuals with suspected IIH. The gene expression signatures found suggest a systemic inflammatory status corresponded by local brain inflammatory processes in subjects with elevated ICP. Putative biomarker candidates are discussed, although further studies are necessary to validate their possible research and clinical use. These findings may have implications in understanding neurophysiologic changes during spaceflight.

\section{RESULTS}

Subject information

Patient information is presented in Table 1 and the Supplementary Table e-1. The average age was 33.8 \pm 9.7 years (range 18-51), 2 males and 20 females. No subject within the lower ICP group was diagnosed with $\mathrm{IIH}$. The subjects in the higher ICP group were diagnosed with $\mathrm{IH}$ and exhibited typical ocular symptoms. No correlation was found between ICP and RNFL thickness (Pearson correlation coefficient between ICP and the average RNFL of both eyes was less than 0.4).

miRNA and mRNA expression in higher and lower ICP subjects In plasma, the average detection rate reached almost $45 \%$ of the targets in the TaqMan Open Array miRNA panel and $75 \%$ for the mRNA Inflammation Open Array. In CSF, the average detection rate for miRNAs was approximately $15 \%$ of the targets and approximately $25 \%$ for mRNA targets. Very few samples (two or less) showed a lower detection rate and those were excluded from analysis. Table 2 lists the differentially expressed miRNAs and mRNAs between subjects in the higher and lower ICP groups, after a Mann-Whitney test was applied with $p<0.05$ (FDR not applied). The sensitivity and reproducibility of the mRNA assay was very high as evaluated by multiple extractions and qRT-PCRs $\left(R^{2}>0.95\right.$ and 0.9 in plasma and CSF samples, respectively).

${ }^{1}$ KBRwyle, NASA Johnson Space Center, Houston, TX, USA; ${ }^{2}$ Exosome Diagnostics, Cambridge, MA, USA and ${ }^{3}$ Baylor College of Medicine, Houston, TX, USA Correspondence: Susana B. Zanello (susana.b.zanello@nasa.gov)

Received: 25 April 2017 Revised: 6 November 2017 Accepted: 13 December 2017

Published online: 11 January 2018 
Table 1. Subject distribution summarizing the mean ICP and standard deviation, ICP range and retinal nerve fiber layer (RNFL) measured by optical coherence tomography (OCT) and averaged between the two eyes on each individual

\begin{tabular}{lll}
\hline & Lower ICP group & Higher ICP group \\
\hline$N$ & 7 & 14 \\
Mean ICP & $14.6 \mathrm{mmHg} \pm 2.5$ & $24.0 \mathrm{mmHg} \pm 4.1$ \\
ICP range & $11.0-17.9 \mathrm{mmHg}$ & $18.8-31.4 \mathrm{mmHg}$ \\
Mean RNFL & $147.6 \mu \mathrm{m} \pm 119.9$ & $195.2 \mu \mathrm{m} \pm 116.3$ \\
\hline $\begin{array}{l}\text { Of 22 subjects recruited, ICP was not able to be determined in one of them } \\
\text { due to technically difficult LP. That subject was therefore not included in } \\
\text { the study and data collection was completed in } 21 \text { subjects. The cut off } \\
\text { between normal and elevated ICP was established at } 18 \mathrm{~mm} \mathrm{Hg}\end{array}$
\end{tabular}

Pathway analysis

miRNA Target Filter analysis in IPA was performed for the complete gene expression data from the miRNA and Inflammation mRNA panels in CSF and plasma. The process allows the combination of experimental gene expression data with the existing knowledge base of predicted and validated miRNA-target relationships. Figure 1 summarizes the result of this process, depicting miRNAs, their targets and the most represented canonical pathways related to inflammation, eicosanoid-phospholipase signaling and immune cell migration.

A comparison was run between the CSF and plasma expression data sets. Of 205 genes with expression data in plasma and 162 genes in CSF, only 31 were represented in both, and only two, miRNA-16 and CD80 (CD86), were differentially expressed in both CSF and plasma (Table 2). miR-9 and miR-16 were upregulated by approximately 4.7 fold in CSF and plasma of higher ICP subjects, and CD80/86 was downregulated by -5.0 fold and -2.5 fold in CSF and plasma, respectively.

\section{DISCUSSION}

This paper reports a gene expression survey of exosomal RNA in CSF and plasma from patients with elevated ICP $(>18 \mathrm{mmHg})$ compared to patients with normal to moderately elevated ICP $(11-18 \mathrm{mmHg})$. All subjects recruited for the study reported to the neurology clinic, therefore none represented a completely "healthy" volunteer and results need to take into account the existence of underlying conditions.

Although this study only examined a limited panel of mRNAs and miRNAs, it is expected that a similar proportion of overlapping genes would have been found with a wider expression screening, like whole genome microarray or RNAseq. In spite of the small overlap in the set of differentially expressed genes from CSF and plasma, the identified pathways associated with increased ICP were similar. Pro-inflammatory pathways such as acute phase response and interleukin signaling were highly represented. Both gene sets suggest that inflammatory processes are acting both locally in the brain as well as systemically, perhaps contributing to IIH pathophysiology.

Pathway analysis at the CSF level also suggested processes of brain cell death, possibly targeted by miR-9. This hypothesis is also supported by evidence that involves miRNA-9 in degenerative diseases, ${ }^{9}$ as well as promoting glial activation via the NFkB pathway. ${ }^{10}$ Interestingly, miR-9 is itself targeted by miR-16, which was also observed to be upregulated in the high ICP group. Circulating miRNAs are increasingly gaining attention as screening biomarkers in central nervous system (CNS) diseases. ${ }^{11}$ In this work, identified candidates warrant monitoring in larger cohort studies to investigate their biomarker value, i.e., miR-16, also investigated as a biomarker for glioblastoma, ${ }^{12}$ and CD86 (CD80),
Table 2. Differentially expressed genes between subjects with normal-to-mildly elevated ICP and with ICP higher than $18 \mathrm{mmHg}$

\begin{tabular}{|c|c|c|c|c|}
\hline \multicolumn{5}{|l|}{ Plasma } \\
\hline miRNA & $\begin{array}{l}U \\
\text { value }\end{array}$ & $\begin{array}{l}p \\
\text { value }\end{array}$ & $\begin{array}{l}\text { Ratio (higher ICP/lower } \\
\text { ICP) }\end{array}$ & $\begin{array}{l}\text { Fold change (log2 } \\
\text { ratio) }\end{array}$ \\
\hline dme-miR-7 & 80 & 0.007 & 29.53 & 4.88 \\
\hline hsa-miR-938 & 78.5 & 0.009 & 48.11 & 5.59 \\
\hline hsa-miR-143 & 76.5 & 0.013 & 579.88 & 9.18 \\
\hline hsa-let-7i & 77 & 0.013 & 16.73 & 4.06 \\
\hline hsa-miR-618 & 14.5 & 0.014 & 0.009 & -6.80 \\
\hline hsa-miR-374 & 76 & 0.015 & 78.96 & 6.30 \\
\hline hsa-miR-657 & 15 & 0.017 & 0.07 & -3.84 \\
\hline hsa-miR-190b & o 15 & 0.017 & 0.003 & -8.38 \\
\hline hsa-miR-10b & 16 & 0.021 & 0.18 & -2.47 \\
\hline hsa-miR-589 & 74 & 0.025 & 16.38 & 4.03 \\
\hline hsa-miR-16-1 & 74 & 0.026 & 20.45 & 4.35 \\
\hline hsa-miR-298 & 18.5 & 0.027 & 0.0001 & -13.29 \\
\hline hsa-miR-551b & 73 & 0.029 & 594.24 & 9.21 \\
\hline Hsa-miR-545 & 73 & 0.031 & 17.08 & 4.09 \\
\hline Hsa-miR-375 & 19 & 0.038 & 0.003 & -8.38 \\
\hline Hsa-miR-10b & 19 & 0.039 & 0.29 & -1.79 \\
\hline Hsa-miR-145 & 71.5 & 0.042 & 30.74 & 4.94 \\
\hline $\begin{array}{l}\text { Hsa-miR-483- } \\
5 p\end{array}$ & 19.5 & 0.043 & 0.4 & -1.32 \\
\hline Hsa-let-7a & 70.5 & 0.044 & 42.02 & 5.39 \\
\hline $\begin{array}{l}\text { Mmu-miR- } \\
187\end{array}$ & 71 & 0.045 & 31.91 & 5.00 \\
\hline Hsa-miR-154 & 20.5 & 0.045 & 0.008 & -6.97 \\
\hline \multicolumn{5}{|l|}{ Gene target } \\
\hline CXCR3 & 10 & 0.0008 & 0.05 & -4.32 \\
\hline TLR3 & 13 & 0.016 & 0.19 & -2.39 \\
\hline LEFTY2 & 70 & 0.02 & 2.64 & 1.4 \\
\hline TNFSF4 & 67.5 & 0.035 & 6.01 & 2.59 \\
\hline CD70 & 17 & 0.038 & 0.12 & -3.06 \\
\hline EREG & 67 & 0.038 & 51.93 & 5.70 \\
\hline CD80 & 17 & 0.038 & 0.17 & -2.56 \\
\hline \multicolumn{5}{|l|}{ CSF } \\
\hline miRNA & $\begin{array}{l}U \\
\text { value }\end{array}$ & $\begin{array}{ll}p & p \\
\text { value }\end{array}$ & $\begin{array}{l}\text { Ratio (higher ICP/ } \\
\text { lower ICP) }\end{array}$ & $\begin{array}{l}\text { Fold change (log2 } \\
\text { ratio) }\end{array}$ \\
\hline hsa-miR-9 & 75.5 & 0.0182 & 24.89 & 4.64 \\
\hline $\begin{array}{l}\text { hsa-miR- } \\
16\end{array}$ & 71 & 0.0452 & 25.89 & 4.69 \\
\hline \multicolumn{5}{|l|}{ Gene target } \\
\hline STAT5B & 70.5 & 0.006 & 106.98 & 6.74 \\
\hline TGM2 & 67.5 & 0.0115 & 58.04 & 5.86 \\
\hline $\begin{array}{l}\text { CD86 } \\
\text { (CD80) }\end{array}$ & 12.5 & 0.018 & 0.03 & -5.06 \\
\hline S100A9 & 62.5 & 0.036 & 91.53 & 6.52 \\
\hline RIPK2 & 16 & 0.048 & 0.11 & 3.18 \\
\hline
\end{tabular}

The differentially expressed miRNAs and mRNAs with $p<0.05$ as determined by the Mann-Whitney test are shown for both plasma and CSF samples. Ratio values that are $<1$ indicate downregulation. The list can be further filtered for $U$ values lesser than the $U$ critical value ( 20 for plasma and 22 for (SF), providing more stringency

which has been implicated in progressive inflammatory myelopathy. ${ }^{13}$

For a long time, the CNS has been considered a site of immune privilege, suffering from costly physiologic consequences if subject to inflammation due to trauma or infection. Currently, this paradigm is being reexamined by work highlighting the 
A

\section{miRNA, Molecular Targets and Pathways in High ICP}

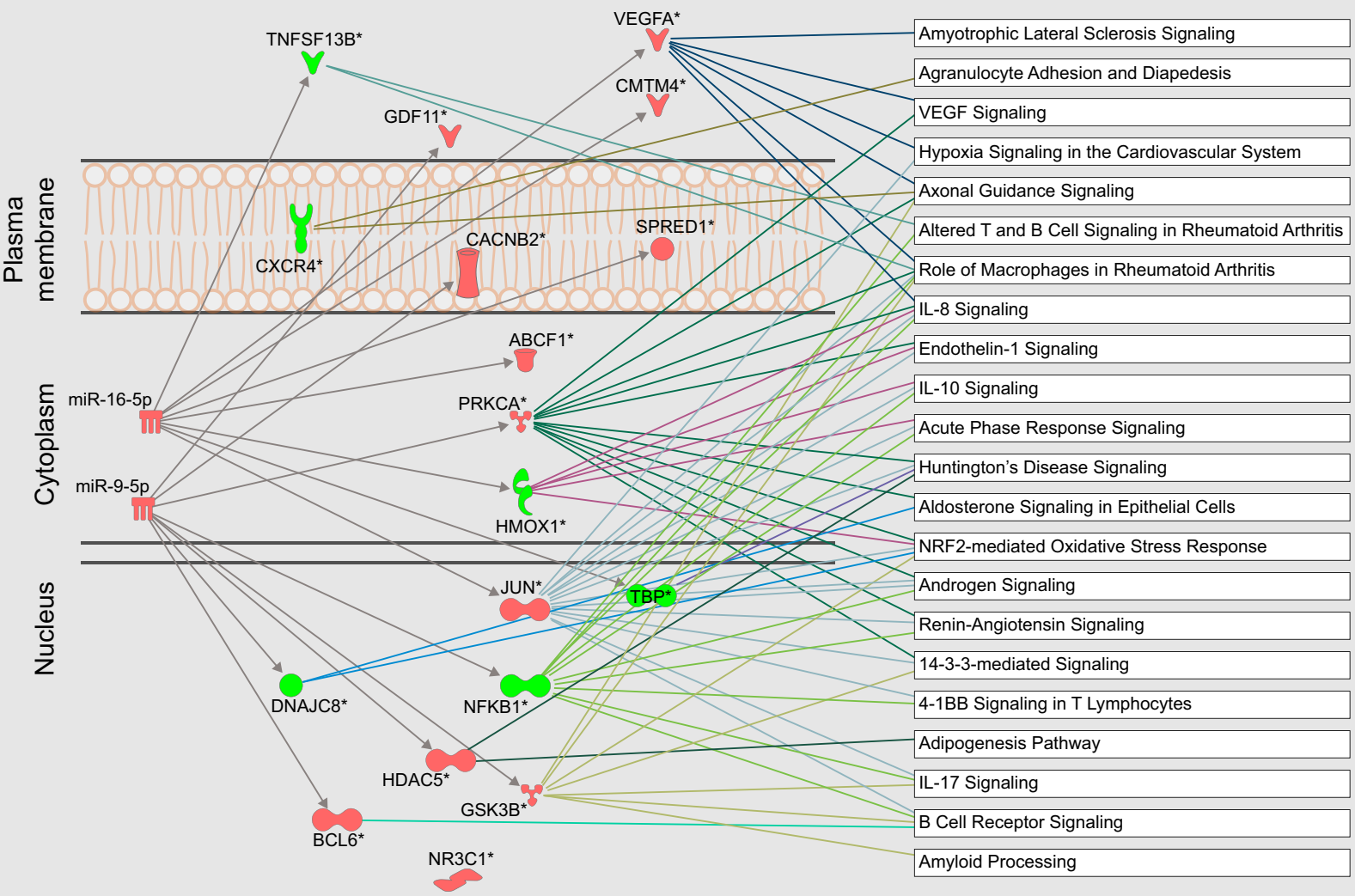

B

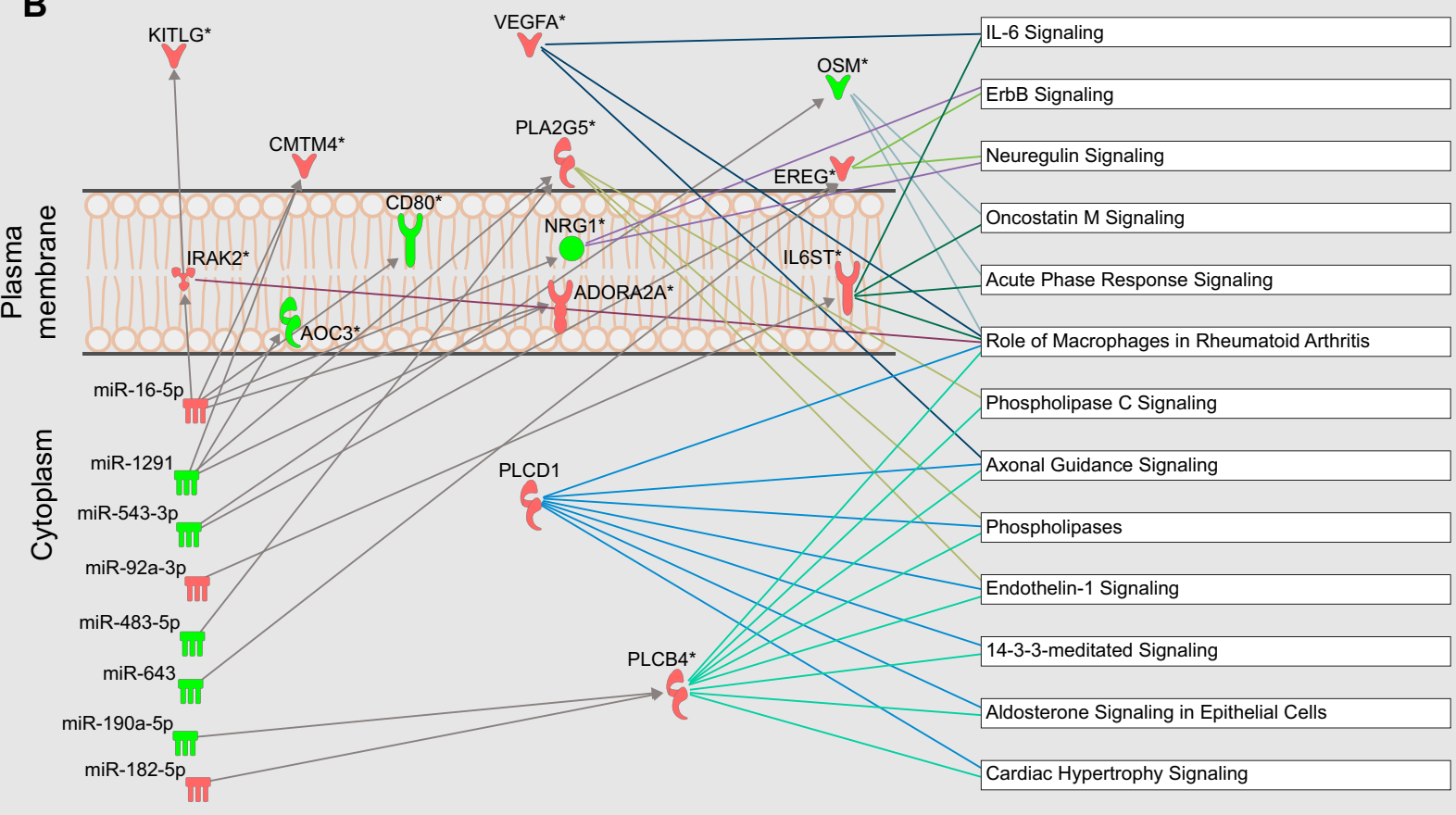

Fig. 1 Main miRNA and canonical pathways represented in patients with elevated ICP. miRNA Target Filter Analysis (Ingenuity Pathways Analysis, IPA ${ }^{\oplus}$, QIAGEN Redwood City, www.qiagen.com/ingenuity) was applied to the differentially expressed gene data set from CSF (a) and plasma (b). miRNA and target relationships are shown by arrows, as well as the main possibly affected canonical pathways 
protective action that a controlled inflammatory response may have on the CNS. For example, it has been shown that mice deficient in functional $T$ cells underperform normal mice in cognitive tasks and this pro-cognitive effect may be mediated by $\mathrm{IL}-4{ }^{14,15}$ Our work supports the existence of an underlying subclinical inflammatory status in $\mathrm{IIH}$. Further studies should be directed towards assessing the extent of these processes and their correlation with neurocognitive parameters.

This study has some limitations. First, it constitutes an exploratory study with small sample size. Second, the lower ICP average, $14.6 \mathrm{mmHg}$, lies on the higher limit of what is considered a normal ICP $(5-15 \mathrm{mmHg})$, and therefore the comparison with high ICP may not yield as many differentially expressed genes as would be expected in comparison with a normal ICP group. Third, IIH patients are arguably a distinct population from the normal healthy astronaut. Since IIH is more prevalent in women, this study contains a majority of females, also deviating from the sample population of astronauts so far screened for VIIP.

Our findings support new concepts regarding the communication between the immune and nervous systems. The IIH patients evidenced signs of generalized inflammation that may be affecting neural physiology at various levels, from neural signaling to cognition. ${ }^{16}$ These patients were predominantly obese (average $\mathrm{BMI}=34.7 \pm 7.4 \mathrm{~kg} / \mathrm{m}^{2}$ ), with likely associated metabolic syndrome, chronic low-grade inflammation, and insulin resistance and other obesity-associated complications, for which pathways and processes were identified in the analysis.

The value of this study predominantly lies in being the first evaluation of the molecular players in $\mathrm{IH}$ and the first comparison between expression profiles from exosomes in CSF and plasma for this condition. Because elevation of ICP is a hallmark of the disease, findings from this study may be relevant to the VIIP syndrome. The results from the CSF survey suggest neuro-immunomodulatory processes, and further work including astronauts exposed to microgravity and the concomitant fluid shifts with ICP increase, is proposed to test this hypothesis on a more relevant sample population.

\section{METHODS}

Sample collection

Methods were performed in accordance with relevant regulations and guidelines. The study was reviewed and approved by the institutional review boards at Johnson Space Center and Baylor College of Medicine $(B C M)$, Houston, TX. Subject recruitment was done at BCM and written informed consent was obtained from all subjects.

The study population was a pool of neurological patients with suspected $\mathrm{IIH}$, requiring diagnostic or therapeutic LP. Subjects were recruited on the basis of the modified Dandy criteria for $\mathrm{IH}$ and the $\mathrm{IH}$ treatment trial, ${ }_{1}^{17}$ consisting in the presence of signs and symptoms of raised ICP $(>20 \mathrm{~cm}$ $\mathrm{H}_{2} \mathrm{O}=14.7 \mathrm{mmHg}$ ), absence of localized findings except those from increased ICP, or abnormal neuroimaging except for empty sella turcica and distended optic nerve sheath. The subject was awake and alert, and no other cause of increased ICP was present. For the LP, the subjects lay in the lateral decubitus position with legs slightly extended. Once the needle was in the thecal sac at the L3-L4 or L4-L5 region, the manometer was observed for the presence of pulse and respiratory waves to indicate patent communication between needle and subarachnoid space. Next, the opening pressure was monitored over a period of $5 \mathrm{~min}$, ICP was measured every minute, and the average calculated. CSF was then drained as per the normal clinical procedure. A volume of $5 \mathrm{ml}$ was collected specifically for the purpose of this study.

Of 22 subjects recruited, ICP was not able to be determined in one of them due to technically difficult LP. That subject was therefore not included in the study and data collection was completed in 21 subjects. The cut off between normal and elevated ICP was established at $18 \mathrm{~mm}$ $\mathrm{Hg}$. Patients were grouped into "normal to moderately high ICP" subjects (less than $18 \mathrm{mmHg}$ ) and high ICP (higher than $18 \mathrm{mmHg}$ ). Seven "normal to mild ICP" and 14 high ICP subjects were compared in the study.
Additionally, subjects had $10 \mathrm{ml}$ blood drawn for plasma separation in $\mathrm{K}_{2}$ EDTA BD Vacutainer blood collection tubes with gel barrier. All plasma and CSF samples were pre-filtered through a $0.8-\mu \mathrm{m}$ syringe filter prior to microvesicular processing, aliquoted and stored at $-80^{\circ} \mathrm{C}$ in a freezer at the Center for Space Medicine at BCM and then shipped to the Exosome Diagnostics facilities in Cambridge, MA.

\section{Exosome and RNA isolation}

CSF at volume of $5 \mathrm{ml}$ and plasma at volume of $4 \mathrm{ml}$ (pre-filtered) were used for exosome isolation by the ExosomeDx spin column-based method (expRNAeasy SerumPlasma Maxi kit, Qiagen, Valencia, CA). The method allows the reproducible isolation of high-quality exosome-specific RNA, which includes both mRNA and miRNA fractions. Briefly, the pre-filtered sample was mixed 1:1 with $2 \times$ binding buffer (XBP) and added to the exoEasy membrane affinity column to bind the exosomes to the membrane. After centrifugation, the flow-through was discarded and wash buffer (XWP) was added to the column to wash off non-specifically retained material. After another centrifugation and discarding of the flowthrough, the vesicles were lysed by adding QIAzol solution and following the addition of chloroform, thorough mixing and centrifugation to separate organic and aqueous phases, the aqueous phase was recovered and mixed with ethanol. The sample-ethanol mixture was added to an RNAeasy MinElute spin column and centrifuged. The column was washed once with buffer RWT, and then twice with buffer RPE followed by elution of RNA in a final volume of $14 \mu \mathrm{L}$ water.

\section{MicroRNA analysis}

Reverse transcription (RT) of miRNA was performed using the Megaplex ${ }^{\mathrm{TM}}$ RT Primers (ThermoFisher Scientific, Waltham, MA) to prepare CDNA for real-time PCR analysis on a TaqMan MicroRNA Array, including a preamplification step. Collectively, the Human Pool Set v3.0 containing two Megaplex TM RT Primer Pools, Pools A v2.1 and B v3.0 cover 754 unique microRNAs. The RT reaction for each primer pool was set as follows: $1 \times$ Megaplex RT Primers (Pool A or B), $2 \mathrm{mM}$ dNTPs (with dTTP), $10 \mathrm{U} / \mu \mathrm{L}$ MultiScribe reverse transcriptase, $1.5 \mathrm{mM} \mathrm{MgCl} 2,1 \times \mathrm{RT}$ buffer, $0.25 \mathrm{U} / \mu \mathrm{L}$ RNAse inhibitor, in a final volume of $15 \mu \mathrm{L}$ per reaction. The reaction was run through the temperature sequence per the manufacturer's instructions.

Preamplification was performed in a $50 \mu \mathrm{L}$ reaction with a $10 \mu \mathrm{L}$ aliquot of the RT reaction as a template, $1 \times$ TaqMan PreAmp Master Mix and $1 \times$ Megaplex ${ }^{\mathrm{TM}}$ PreAmp Primers (Pool A or Pool B), in a temperature cycling (16 cycles) according to the manufacturer. Pre-amplified samples were diluted 1:10 in 0.1× TE pH 8.0. The TaqMan OpenArray Human MicroRNA Panel was used for final PCR detection.

In this final step, the DNA polymerase of the TaqMan Universal PCR Master Mix amplifies the specific cDNA targets using sequence-specific primers and probe on the TaqMan microRNA Array. $25 \mu \mathrm{L}$ TaqMan OpenArray ${ }^{\circ}$ Real-Time PCR Master Mix and $13 \mu \mathrm{L} 0.1 \times$ buffer TE pH 8.0 were loaded into each of two adjacent wells per sample on a clean 96-well plate. For each sample, $12 \mu \mathrm{L}$ of diluted Pool A PreAmp was pipetted into one well of each pair and $12 \mu \mathrm{L}$ of diluted Pool B PreAmp was pipetted into the other well for a total volume of $50 \mu \mathrm{L}$ in each well. For each sample and primer pool set (Pool A or Pool B), $5 \mu \mathrm{L}$ of each reaction mixture was pipetted into each of eight wells on an OpenArray 384-well sample plate. Samples were loaded onto OpenArray plates using the standard Accufill ${ }^{\mathrm{TM}}$ protocol. Amplification was performed according to the protocol established for the TaqMan OpenArray Human MicroRNA panel.

\section{mRNA analysis}

The RT reaction was carried out in a final volume of $20 \mu \mathrm{L}$ containing: $14 \mu \mathrm{L}$ RNA sample, $2 \mu \mathrm{L}$ Superscript reverse transcriptase enzyme mix, and $4 \mu \mathrm{L}$ VILO Master Mix (which includesRNaseOUT ${ }^{\mathrm{TM}}$ Recombinant Ribonuclease Inhibitor, $\mathrm{MgCl} 2$, dNTPs and random primers). The reaction proceeded for $10 \mathrm{~min}$ at $95^{\circ} \mathrm{C}, 15 \mathrm{~s}$ at $95^{\circ} \mathrm{C}, 4 \mathrm{~min}$ at $60^{\circ} \mathrm{C}$, followed by inactivation at $95^{\circ}$ C for 10 min.

Pre-amplification was done using Human Inflammation PreAmp Primers (Pool A or B) $5 \mu \mathrm{L}$, TaqMan PreAmp Master Mix $10 \mu \mathrm{L}$, and $5 \mu \mathrm{L}$ of RT product (CDNA) in a final volume of $50 \mu \mathrm{L}$. The temperature sequence was the following: $10 \mathrm{~min}$ at $95^{\circ} \mathrm{C}, 15 \mathrm{~s}$ at $95^{\circ} \mathrm{C}$ and $4 \mathrm{~min}$ at $60^{\circ} \mathrm{C}$ (for 14 cycles), followed by inactivation at $95^{\circ} \mathrm{C}$ for $10 \mathrm{~min}$. The pre-amplified samples were stored at $4{ }^{\circ} \mathrm{C}$ until the PCR was performed.

The TaqMan OpenArray Human Inflammation Panel (Applied Biosystems, ThermoFisher Scientific) was used for final PCR detection. These 
contain TaqMan gene expression assays dried down in 384-well TaqMan Array microfluidic cards targeting pathway relevant gene targets. The panel covers 586 genes that have been studied as targets for a range of inflammatory diseases, plus 21 endogenous control genes.

PreAmp products for each sample were mixed and diluted 1:10 with $0.1 \times$ $\mathrm{TE} \mathrm{pH}=8.0$. For each sample, $35 \mu \mathrm{L}$ of $2 \times$ TaqMan $^{\circ}$ OpenArray Real-Time PCR Master Mix was mixed with $35 \mu \mathrm{L}$ mixed, diluted PreAmp. $5 \mu \mathrm{L}$ of each reaction mixture was pipetted into each of 12 wells on an OpenArray 384well sample plate. Samples were loaded onto OpenArray plates using the standard Accufill ${ }^{\mathrm{TM}}$ protocol. Amplification was performed according to the protocol established for the TaqMan OpenArray Human Inflammation Panel with the QuantStudio ${ }^{\mathrm{TM}} 12 \mathrm{~K}$ Flex Real-time PCR system.

\section{Data analysis and bioinformatics}

The mRNA data comprises cycle threshold $(\mathrm{Ct})$ values from the OpenArray QuantStudio $^{\mathrm{TM}} 12 \mathrm{~K}$ Flex Real-time PCR system, which is converted to gene expression levels for a panel of 607 inflammation-related genes and endogenous control genes. The miRNA data set consists of 754 different miRNAs. Both data sets were processed using the $R$ statistical programming environment. Missing data points were assigned 'undetermined', indicating sequence abundances below the detection limit of the OpenArray platform. Quantile normalization was applied to data sets to adjust for technical variation and to make data points comparable among each other. Features or targets with greater than 10 undetermined values were removed from the analysis. An additional filtering step was performed to remove features showing little or no variation across samples since these targets with relatively constant $\mathrm{Ct}$ levels are less likely to be differentially expressed. This was achieved removing features with an interquartile range of $<2$. A Mann-Whitney test was performed to compare the samples from patients with elevated and normal ICP. Profiling hundreds of genes at the same time represents a large multiple hypothesis-testing problem from the statistical perspective. While typically this is adjusted by computing false discovery rates (FDR), in this case, however, due to the low number of samples, the multiple testing correction was not applied (since none of the targets showed less than $15 \%$ FDR)

Once the set of differentially expressed genes was obtained, functional analyses were generated through the use of QIAGEN's Ingenuity Pathways Analysis (IPA', QIAGEN Redwood City, www.qiagen.com/ingenuity). First, the predicted targets for the differentially expressed miRNAs were identified using miRNA Filter Analysis, which relies on the Ingenuity knowledge database of reported miRNA targets. This allowed the generation of a comprehensive list of fold-change expression values for miRNA and mRNA, which was then subjected to core analysis.

\section{Data availability}

Raw data supporting the results reported in this article can be found in the NASA Life Sciences Data Archive (https://lsda.jsc.nasa.gov/lsda_home.aspx)

\section{ACKNOWLEDGEMENTS}

This material is based upon work supported by the National Aeronautics and Space Administration under Grant/Contract/Agreement No NNX15AW48G to SZ. We would like to express our thanks to Drs. R. Tang and B. Gutierrez Flores from the University of Houston College of Optometry for performing the ophthalmological evaluations, and John Blackwell, USRA-LPI, for artwork. This study was funded by NASA (Grant NNX15AW48G). Other Contributors (non-authors): Rosa Tang, MD, MPH (University Eye Institute, University of Houston; ophthalmic examinations); B Gutierrez Flores, MD (University Eye Institute, University of Houston; ophthalmic examinations)

\section{AUTHOR CONTRIBUTIONS}

S.Z., study concept and design, study supervision, analysis and interpretation of data, manuscript writing, funded investigator. V.T., gene expression data and statistical analysis. J.H., sample processing and analysis. J.S., study supervision, concept and design, data analysis. E.C., research coordination. B.S., sampling handling, subject data analysis. E.B., sample collection, study concept and design, critical revision of manuscript.

\section{ADDITIONAL INFORMATION}

Supplementary information accompanies the paper on the npj Microgravity website (https://doi.org/10.1038/s41526-017-0036-6).
Competing interests: Dr Zanello is the recipient of the NASA award funding this study, as well as other research support from the agency. Dr Tadigotla is employed by Exosome Diagnostics, Inc. and holds stock options from Exosome Diagnostics. Dr James Hurley is employed by Exosome Diagnostics, Inc. and holds stock options from Exosome Diagnostics. Dr Skog is Chief Scientific Officer at Exosome Diagnostics, Inc and holds stock options from Exosome Diagnostics. Mr Brian Stevens and Ms Eusebia Calvillo report no disclosures. Dr Bershad performs neurology clinical practice, and conducts several research projects funded by NASA and the NSBRI (National Space Biomedical Research Institute)

Publisher's note: Springer Nature remains neutral with regard to jurisdictional claims in published maps and institutional affiliations.

\section{REFERENCES}

1. Mader, T. H. et al. Optic disc edema, globe flattening, choroidal folds, and hyperopic shifts observed in astronauts after long-duration space flight. Ophthalmology 118, 2058-2069 (2011).

2. Peng, K. P., Fuh, J. L. \& Wang, S. J. High-pressure headaches: idiopathic intracranial hypertension and its mimics. Nat. Rev. Neurol. 8, 700-710 (2012).

3. Sorensen, P. S., Thomsen, A. M. \& Gjerris, F. Persistent disturbances of cognitive functions in patients with pseudotumor cerebri. Acta Neurol. Scand. 73, 264-268 (1986).

4. Kharkar, S. et al. Cognitive impairment in patients with pseudotumor cerebri syndrome. Behav. Neurol. 24, 143-148 (2011).

5. Paloski, W. et al Risk of sensory-motor performance failuresaffecting vehicle control during space missions: a review of the evidence. J. Gravit. Physiol. 15, 1-29 (2008).

6. Young, L. R. Vestibular reactions to spaceflight: human factors issues. Aviat. Space Environ. Med. 71, A100-A104 (2000).

7. Strangman, G. E., Sipes, W. \& Beven, G. Human cognitive performance in spaceflight and analogue environments. Aviat. Space Environ. Med. 85, 1033-1048 (2014)

8. Vein, A. A., Koppen, H., Haan, J., Terwindt, G. M. \& Ferrari, M. D. Space headache: a new secondary headache. Cephalalgia 29, 683-686 (2009).

9. Saito, Y. \& Saito, H. MicroRNAs in cancers and neurodegenerative disorders. Front Genet. 3, 194 (2012).

10. Yao, $\mathrm{H}$. et al. MiR-9 promotes microglial activation by targeting MCPIP1. Nat Commun. 5, 4386 (2014).

11. Sheinerman, K. S. \& Umansky, S. R. Circulating cell-free microRNA as biomarkers for screening, diagnosis and monitoring of neurodegenerative diseases and other neurologic pathologies. Front. Cell. Neurosci. 7, 150 (2013).

12. D'Urso Ivo, D. U. F., Damiano, G., Mezzolla, V., Storelli, C. \& Marsigliante, S. miR-15b and miRNA-21 as circulating biomarkers for diagnosis in glioma. Curr. Genom. 16, 304-311 (2015).

13. Menezes, S. M. et al. CD80+ and CD86+ B cells as biomarkers and possible therapeutic targets in HTLV-1 associated myelopathy/tropical spastic paraparesis and multiple sclerosis. J. Neuroinflamm. 11, 18 (2014).

14. Kipnis, J., Cohen, H., Cardon, M., Ziv, Y. \& Schwartz, M. T cell deficiency leads to cognitive dysfunction: implications for therapeutic vaccination for schizophrenia and other psychiatric conditions. Proc. Natl. Acad. Sci. USA 101, 8180-8185 (2004).

15. Derecki, N. C. et al. Regulation of learning and memory by meningeal immunity: a key role for IL-4. J. Exp. Med. 207, 1067-1080 (2010).

16. Pavlov, V. A. \& Tracey, K. J. Neural circuitry and immunity. Immunol. Res. 63, 38-57 (2015).

17. The NORDIC Idiopathic Intracranial Hypertension Study Group Writing Committee. Effect of Acetazolamide on visual function in patients with idiopathic intracranial hypertension and mild visual lossthe idiopathic intracranial hypertension treatment trial. JAMA 311, 1641-1651 (2014).

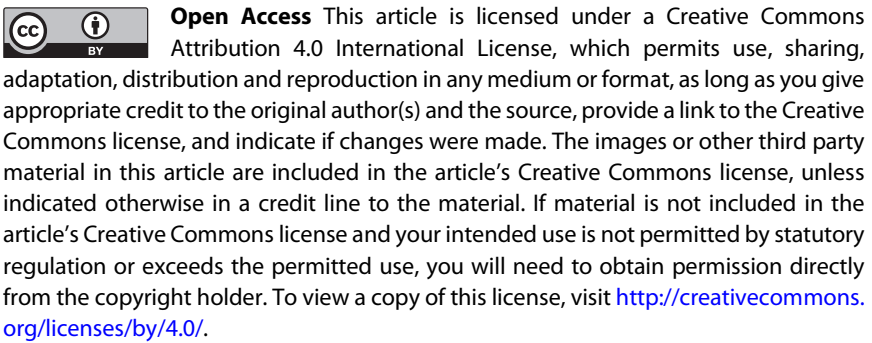

(c) The Author(s) 2018 